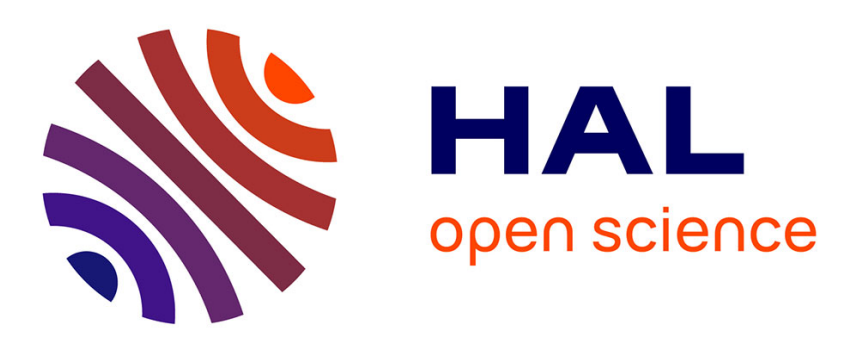

\title{
Plasmon-enhanced diffraction in nanoparticle gratings fabricated by in situ photo-reduction of gold chloride doped polymer thin films by laser interference patterning
}

E. Nadal, Noémi Barros, H. Glénat, J. Laverdant, D. Schmool, H. Kachkachi

\section{To cite this version:}

E. Nadal, Noémi Barros, H. Glénat, J. Laverdant, D. Schmool, et al.. Plasmon-enhanced diffraction in nanoparticle gratings fabricated by in situ photo-reduction of gold chloride doped polymer thin films by laser interference patterning. Journal of Materials Chemistry C, 2017, 5 (14), pp.3553-3560. 10.1039/C7TC00061H . hal-02369460

\author{
HAL Id: hal-02369460 \\ https://hal.science/hal-02369460
}

Submitted on 19 Nov 2019

HAL is a multi-disciplinary open access archive for the deposit and dissemination of scientific research documents, whether they are published or not. The documents may come from teaching and research institutions in France or abroad, or from public or private research centers.
L'archive ouverte pluridisciplinaire HAL, est destinée au dépôt et à la diffusion de documents scientifiques de niveau recherche, publiés ou non, émanant des établissements d'enseignement et de recherche français ou étrangers, des laboratoires publics ou privés. 


\section{Journal of \\ Materials Chemistry C}

\section{Accepted Manuscript}

This article can be cited before page numbers have been issued, to do this please use: E. Nadal, N. Barros, H. GLENAT, J. Laverdant, D. S. Schmool and H. Kachkachi, J. Mater. Chem. C, 2017, DOI:

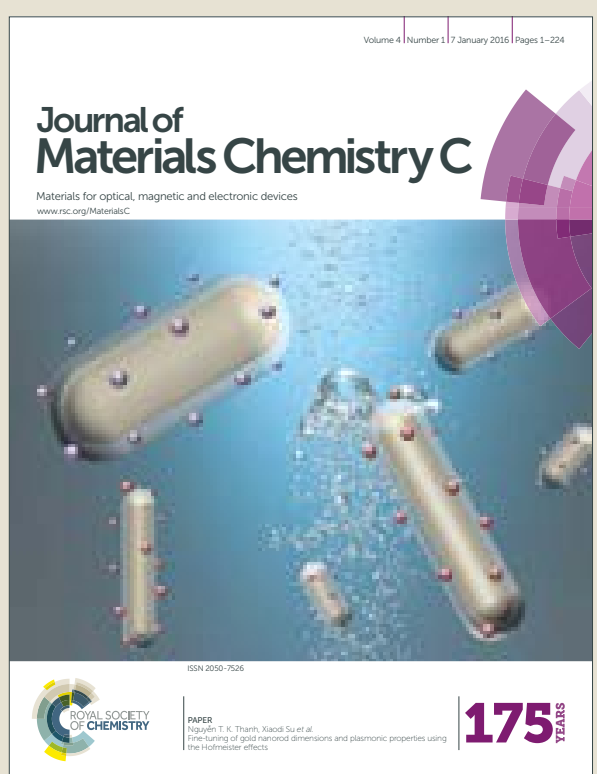

This is an Accepted Manuscript, which has been through the Royal Society of Chemistry peer review process and has been accepted for publication.

Accepted Manuscripts are published online shortly after acceptance, before technical editing, formatting and proof reading. Using this free service, authors can make their results available to the community, in citable form, before we publish the edited article. We will replace this Accepted Manuscript with the edited and formatted Advance Article as soon as it is available.

You can find more information about Accepted Manuscripts in the author guidelines.

Please note that technical editing may introduce minor changes to the text and/or graphics, which may alter content. The journal's standard Terms \& Conditions and the ethical guidelines, outlined in our author and reviewer resource centre, still apply. In no event shall the Royal Society of Chemistry be held responsible for any errors or omissions in this Accepted Manuscript or any consequences arising from the use of any information it contains. 


\title{
Journal of Material Chemistry C
}

\section{ARTICLE}

\section{Plasmon-enhanced diffraction in nanoparticle gratings fabricated by in-situ photo-reduction of gold chloride doped polymer thin films by laser interference patterning}

Received 00th January 20xx Accepted 00th January 20xx

DOI: $10.1039 / \times 0 \times x 00000 x$

www.rsc.org/

\author{
E. Nadal ${ }^{a, b}$, N. Barros ${ }^{a, b}$, H. Glénat ${ }^{a}$, J. Laverdant ${ }^{c}$, D.S. Schmool ${ }^{d}$, H. Kachkachi ${ }^{a, b}$
}

\begin{abstract}
We propose a new method for light-induced in situ synthesis of gold nanoparticle gratings in polymer thin films. The polymer films are doped with gold precursors and then irradiated with an interference pattern leading to the spatially controlled photo-reduction of metallic ions. An annealing procedure is then used to induce nanoparticles growth while maintaining the spatial organization within the film. The originality of the method resides in the fact that we use a continuous low-power laser excitation in the visible range, which opens the way to large-area manufacturing processes. In terms of optical properties, such fabricated gold nanoparticle gratings exhibit both plasmonic and diffractive properties with plasmon enhanced diffraction efficiency.
\end{abstract}

\section{Introduction}

The development of metal-dielectric nanocomposite materials with plasmonic properties is of great interest for many applications such as sensing ${ }^{1}$, SERS ${ }^{2}$, photonics ${ }^{3}$ and photovoltaics ${ }^{4,5}$. In particular, it has been shown that plasmonic nanoparticle gratings (PNG) can offer new detection schemes in bio-sensing and can significantly improve the detection sensitivity ${ }^{6-8}$. For this purpose, polymers, as host matrices, offer several advantages in terms of processing, optical transparency and low cost. In addition, they are suitable for single-step nanofabrication approaches such as in situ photo-reduction and direct laser patterning.

Regarding the light-controlled formation of organized assemblies of nanoparticles in polymer matrices, several approaches have been studied. One possibility consists in adding previously synthesized nanoparticles in azobenzenebased photosensitive polymers, which are widely used in the field of optical data storage. Several authors have used a standard interferometry patterning technique to periodically structure the polymer surface by photo-isomerization ${ }^{9}$, thus inducing a nanoparticle distribution ${ }^{10-12}$. This method allows for a relatively easy control of the spatial periodicity of the pattern. In the case of gold nanoparticles, it has been shown, however, that the polymer structuring properties tend to rapidly deteriorate when the nanoparticles concentration increases

\footnotetext{
a.PROMES, CNRS (UPR8521), Rambla de la thermodynamique, 66100, Perpignan, France.

b. University of Perpignan Via Domitia (UPVD), 52 Avenue Paul Alduy, 66100 Perpignan, France.

Univ Lyon, Université Claude Bernard Lyon 1, CNRS, Institut Lumière Matière, F69622, VILLEURBANNE, France.

d. GEMaC, CNRS (UMR 8635), Université de Versailles/Saint-Quentin, Université Paris-Saclay, 45 Avenue des Etats-Unis, 78035 Versailles, France.

Email-elie.nadal@univ-perp.fr
}

over $0,1 \mathrm{w}^{13}$. This constitutes a limit to the control of the intrinsic optical properties of the composites and to the development of plasmon-resonance based applications. Moreover, surfactants must be added at the surface of the nanoparticles to avoid aggregation, which sometimes requires complex chemical treatments.

Another interesting approach that has been explored is the in situ reduction of metallic precursors in polymer films. In particular, the in situ formation of gold nanoparticles induced by UV irradiation and/or annealing has been extensively studied in polyvinyl alcohol (PVA) and has been shown to lead to uniformly distributed nanoparticles with controlled size and shape ${ }^{14-17}$. In order to control the spatial distribution of the nanoparticles, it is possible to perform a spatially controlled photo-reduction of the precursor, which can be achieved by controlling the light intensity distribution. This can be done by using laser interference patterning techniques which are very versatile in terms of irradiation pattern ${ }^{18}$ and are suitable for large-area fabrication processes. For gold nanoparticle composites, only a few studies have been reported so far ${ }^{19,20}$. In these studies, pulsed lasers have been used. In this case, the in situ photo-reduction of the gold precursor has been explained by a two-photon absorption mechanism. However, such nonlinear absorption processes ${ }^{21}$ can only be achieved using pulsed lasers as they require high powers. To reach the desired power, the beams must be focused and this limits the size of the irradiated area to hundreds of $\mu \mathrm{m}^{2}$. Another original fabrication technique that combines both the use of azobenzene-based photosensitive polymers and UV in situ photo-reduction to synthesize plasmonic-photonic crystals from silver nanoparticle arrays has also been reported ${ }^{22}$.

In the present work, we use a continuous laser irradiation at 473 $\mathrm{nm}$ to perform the in situ photo-reduction of metallic precursors in a PVA thin film using an interference pattern. To the best of our knowledge, it is the first time that photo- 
reduction is shown to operate in the visible range with a continuous laser and without the use of photosensitizer to initiate the reaction. The photo-reduction is followed by an annealing protocol that allows for the nanoparticle nucleation and growth. We observe a significant difference in the PNG we obtain as compared with those obtained with pulsed laser patterning, which is the formation of a surface relief grating after nanoparticle growth. As will be seen below, such fabricated PNG present unusual optical properties such as plasmon-enhanced diffraction ${ }^{23}$.

This article is organized as follows: In the next section we describe the fabrication technique and the experimental setups. In Section 3, we discuss our results focusing on structural and optical properties of the fabricated PNG. The last section presents our conclusions and perspectives.

\section{Experimental}

We now briefly describe our method first focusing on the chemical mechanisms and treatment by irradiation. Fig. 1 summarizes the main fabrication steps. Polymer films doped with gold precursors are prepared by spin coating. These films are then irradiated with an interference pattern generated by a laser interferometry setup. The light allows for the in situ photoreduction of the gold ions. The samples are then annealed to generate the nanoparticle formation from reduced gold atoms. We have used $\mathrm{HAuCl}_{4}: 3 \mathrm{H}_{2} \mathrm{O}(99,99 \%$ pure, Aldrich) as a metallic precursor and Polyvinyl Alcohol (PVA, average molecular weight $12000,86 \%$ hydrolyzed, Aldrich) as a polymer. The solution used for spin coating is prepared in a two-step process. We first dissolve $600 \mathrm{mg}$ of PVA in $3 \mathrm{~mL}$ of deionized water. The solution is then stirred and heated at $60^{\circ} \mathrm{C}$ over night to reach complete dissolution. We then dissolve $47 \mathrm{mg}$ of $\mathrm{HAuCl}_{4}: 3 \mathrm{H}_{2} \mathrm{O}$ in $3 \mathrm{~mL}$ of the PVA solution so as to obtain 5\%w Au/PVA composite after solvent evaporation.
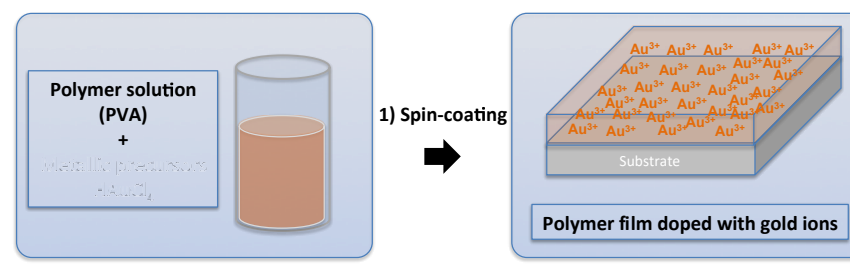

Polymer film doped with gold ions

2) Irradiation

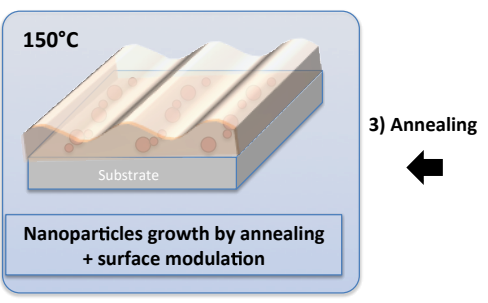

Mach-Zehnder interferometer

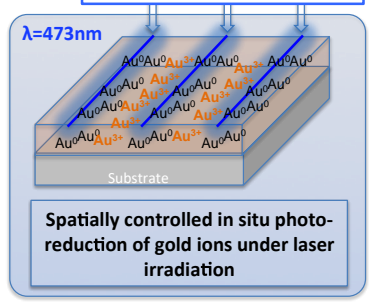

Figure 1: Main steps of the fabrication technique.
The blend thus obtained is stirred in the dark for 1 hour and then filtered with $5 \mu \mathrm{m}$ pores before spinPedating? spin first at $1500 \mathrm{rpm}$ for $60 \mathrm{~s}$ and then at $5000 \mathrm{rpm}$ for $5 \mathrm{~s}$. We use glass substrates $(25 \mathrm{~mm} \times 25 \mathrm{~mm}$ ) that have previously been cleaned with successive ultrasonic baths of acetone, isopropanol and distilled water and finally dried with nitrogen. The thickness of the films is measured with a profilometer (Dektak) and is around $1 \mu \mathrm{m}$. Undoped PVA films with equivalent thickness are also prepared as a reference. The films are then kept in the dark at room temperature for two days until the solvent evaporation is complete. We used this procedure instead of a classic annealing in order to remove the remaining solvent because such treatment would induce the thermal reduction of the precursor and nanoparticle growth ${ }^{14}$. The stability of the precursor/polymer film composite has been guaranteed by monitoring the gold ions concentration just after deposition and again two days later. No significant changes were observed. Performing measurement after longer periods of time (up to 2 weeks) after spin-coating does not bring about any further changes in the samples regarding their reaction to subsequent treatments. Hence, we believe this procedure is adequate for "drying out" the precursor/polymer composite. Regarding the gold/polymer ratio, the value of $5 \% \mathrm{w}$ has been adopted since it leads to a nanocomposite film with easily measurable plasmonic properties. Furthermore, we have also found that only gold/polymer ratios around $5 \% \mathrm{w}$ render a significant surface modulation in the final plasmonic gratings. Finally, with the help of the protocol described here, larger gold/polymer ratios are likely to lead to the formation of larger nanoparticles or aggregates thereof.

The films are then irradiated with an interference pattern to induce a spatially controlled photo-reduction of the gold ions. We have used a Mach-Zehnder type interferometer with a laser at $473 \mathrm{~nm}$ (Cobolt Blues, $50 \mathrm{~mW}$ ) to generate a periodic irradiation pattern, see Fig. 2. The beam is expanded and polarized before passing through a beam splitter. The two beams are then redirected by two mirrors so as to interfere at the sample surface with a given incidence angle. In this study, the grating spacing is set to $2 \mu \mathrm{m}$ but can easily be varied by adjusting the angle of incidence $\theta$ of the interfering beams ${ }^{24}$. The grating period $\Lambda$ is given by

$$
\Lambda=\frac{\lambda}{2 \sin (\theta)}(1)
$$

where $\lambda$ is the incident light wavelength. The light flux is set at $100 \mathrm{~mW} / \mathrm{cm}^{2}$ and the polarization is set so that the beams impinge on the sample in the $s$ configuration. In a typical experiment, a sample is irradiated from 5 to $60 \mathrm{~min}$. When the irradiation begins, a diffraction grating is formed in the polymer composite film by the interference pattern. We then measure the intensity of the first-order diffracted beam during the irradiation to follow the grating formation in real time. To monitor the grating formation, we use a second laser (HeNe laser at $633 \mathrm{~nm}, 5 \mathrm{~mW}$ ) with a fluence of $1 \mathrm{~mW} / \mathrm{cm}^{2}$ that impinges on the sample at normal incidence and is diffracted by the grating. 


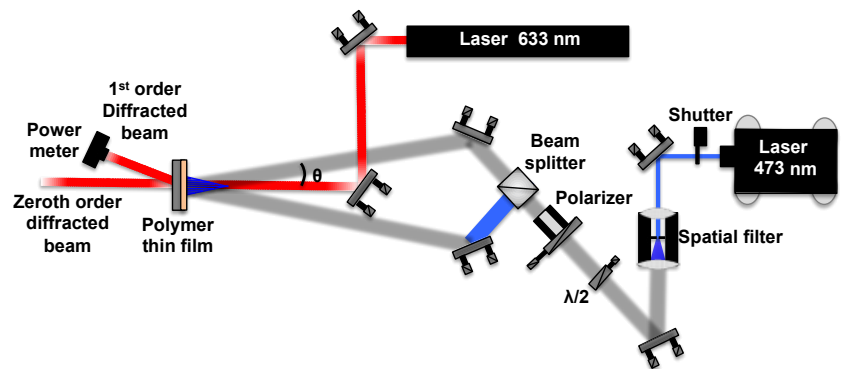

Figure 2: Mach-Zehnder interferometer setup with $1^{\text {st }}$ order diffracted beam measurement

We then measure the intensity of the first-order diffracted beam during the irradiation to follow the grating formation in real time. Monitoring the diffraction efficiency allows us to evaluate the optimum irradiation time as well as to probe the dynamics of the PNG formation.

After irradiation, the gold ions in the polymer matrix have been photo-reduced into gold atoms. Then, a final annealing step is used to trigger the formation of nanoparticles. The samples are annealed on a hot plate. The temperature at the sample surface, controlled with a thermocouple, is progressively increased from room temperature to $100^{\circ} \mathrm{C}$ during $2 \mathrm{~min}$ and then kept at $100^{\circ} \mathrm{C}$ for $1 \mathrm{~min}$. These conditions have been chosen based on previous studies of annealed $\mathrm{HAuCl}_{4} / \mathrm{PVA}$ nanocomposites ${ }^{14}$ and on our own experience. In this last step, we observe that the sample color turns to purple/red and a surface relief grating appears, clearly visible to the eye. The surface topography of the films obtained after annealing has been characterized by AFM measurements (Model SMENA, NTMDT), as shown in Fig.7. The sample thicknesses are evaluated by performing a scratch on the polymer film, whose depth is then measured with a profilometer (DEKTAK XT, Bruker).

The PNG are further characterized by optical spectroscopy before and after irradiation (see Fig.6), and also after annealing (see Fig.8). For this purpose, we have built two experiments for the acquisition of: (1) extinction spectra for determining the plasmonic properties of the gold nanoparticle assemblies and (2) angle resolved spectra that permit the study of the diffractive properties of the PNG.

Experiment (1), whose aim is to measure extinction spectra on localized areas of our samples, is illustrated in Fig. 3. We use a UV-Visible light source (DH2000, Ocean Optics) collimated via a parabolic mirror. The beam is then redirected by a $50: 50$ beam splitter and focused on the sample using a microscope objective (Olympus, $x 40, N A=0,6$ ). The spot size has a diameter of $60 \mu \mathrm{m}$ on the sample. We then use the reflected light to image the area with a CCD camera and the transmitted light is measured with a spectrophotometer for spectral acquisition. To simplify the interpretation of the spectra, the light is only collected at small angles so as to minimize incidence-angle effects and we measure only the zero order beam.

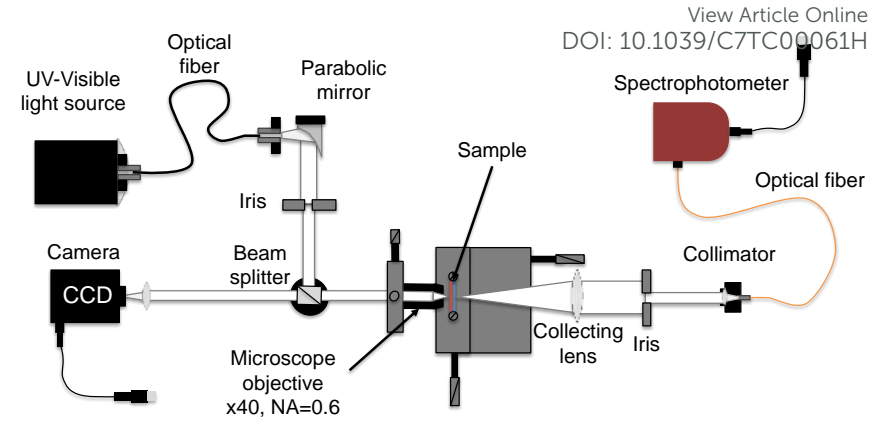

Figure 3: Experiment (1), homemade spectroscopic setup for extinction spectra measurements and imaging through a microscope objective.

Finally, experiment (2) has been developed for the measurement of the spectral dependence of the grating diffraction. The setup is shown in Fig. 4. Again, we use a UVvisible light source that is collimated and polarized. The transmitted light is diffracted by the sample and we observe the different diffracted order beams as well as the angular wavelength dispersion within each diffracted order. The light is collected through a slit and a fiber collimator placed on the moving arm of the goniometer. The collected light is then sent to the spectrophotometer. This allows for the acquisition of the transmittance spectra at different angles (denoted by $\alpha$ in Fig. 4.) and thereby the study of the spectral dependence of the diffraction. We can reconstruct diffraction efficiency maps that reveal how the diffracted intensity varies as a function of the transmitted angle and wavelength. We use the zerothtransmitted order for the normalization such that we measure the diffraction efficiency (see below). The light polarization direction was set parallel to the grating lines such that all diffraction measurements were performed in the TE configuration.

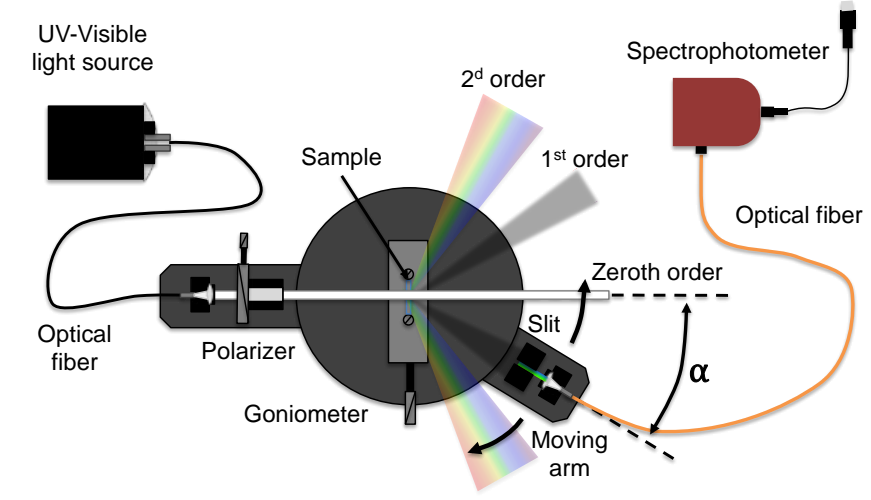

Figure 4: Experiment (2), homemade spectro-goniometer for measuring angular resolved spectra.

\section{Results and discussion}

We first irradiate the film for 60 minutes in order to determine, over time, the different stages of the grating formation. The time evolution of the diffraction efficiency during irradiation is shown in Fig.5. We see that the diffraction starts to increase a few minutes after the irradiation has begun. The increase is slow at the beginning and then becomes almost 
linear until it reaches a maximum. Under the experimental conditions explained earlier, the maximum was reached after approximately $18 \mathrm{~min}$ of irradiation. The diffraction then starts to decrease towards zero after approximately $40 \mathrm{~min}$ of irradiation. We note, however, that the diffraction efficiency slowly increases again if we keep on irradiating.

We have measured UV extinction spectra to verify the laserinduced photo-reduction of the gold ions. For this, we selected three different areas of the same sample: the first area was not irradiated, the second one (referred as (a) in the following) was irradiated for $18 \mathrm{~min}$ to reach the maximum diffraction efficiency and the third one (referred as (b)) was irradiated for $60 \mathrm{~min}$. We have also performed measurements on a sample prepared under exactly the same conditions but irradiated for 60 min with a Gaussian beam at the same intensity (referred as (c)). We use a glass substrate as a reference for all measurements. The results are given in Fig. 6 . We see that the non-irradiated sample presents an extinction band around 325 $\mathrm{nm}$ that corresponds to the ligand-to-metal charge transfer of the $\mathrm{AuCl}_{4}$ - ions ${ }^{25,26}$. This peak is strongly attenuated after $18 \mathrm{~min}$ of irradiation and drastically reduced after $60 \mathrm{~min}$. There is no significant difference between the samples irradiated for $60 \mathrm{~min}$ with the interference pattern or with a Gaussian beam. We thus find that the photo-reduction is almost complete after $60 \mathrm{~min}$, whereas the maximum of diffraction efficiency is reached after 18 min of irradiation, even if there are still gold ions in the film. Furthermore, we note that no peak is observed in the 500-550 $\mathrm{nm}$ range, which corresponds to the gold plasmon resonance wavelength. This shows that no nanoparticles are formed at this stage of the process. However, small gold clusters, that do not develop a measurable plasmon resonance might be formed ${ }^{27}$.

The diffraction efficiency profile can be interpreted as follows. When the irradiation begins, the gold ions start to be photoreduced in the bright fringes. As a consequence, a periodic modulation of the ionic density is created, which is reflected in the refractive index. Therefore, a refractive index grating that diffracts light is formed. As the irradiation continues, the ionic density modulation increases leading to an increase in the diffraction efficiency. The curve reaches a maximum after 18 min of irradiation, which corresponds to the maximal refractive index modulation. After this point, the diffraction efficiency decreases since the ions start to be photo-reduced in the dark fringes, leading to a drop in the ionic concentration in these zones, and a decrease of the refractive index modulation. The evolution of the gold atom concentration during irradiation is schematically illustrated in Fig. 5(b). In addition, some ion diffusion may also occur, leading to a homogenization of the ionic concentration ${ }^{28}$. Finally, the gold atom distribution becomes almost uniform as all gold ions are reduced and the diffraction goes to a minimum after $40 \mathrm{~min}$ of irradiation. As irradiation continues, we observe a new gradual increase of the diffraction. We interpret this phenomenon as the formation of small gold clusters in the bright fringes by aggregation of gold atoms.

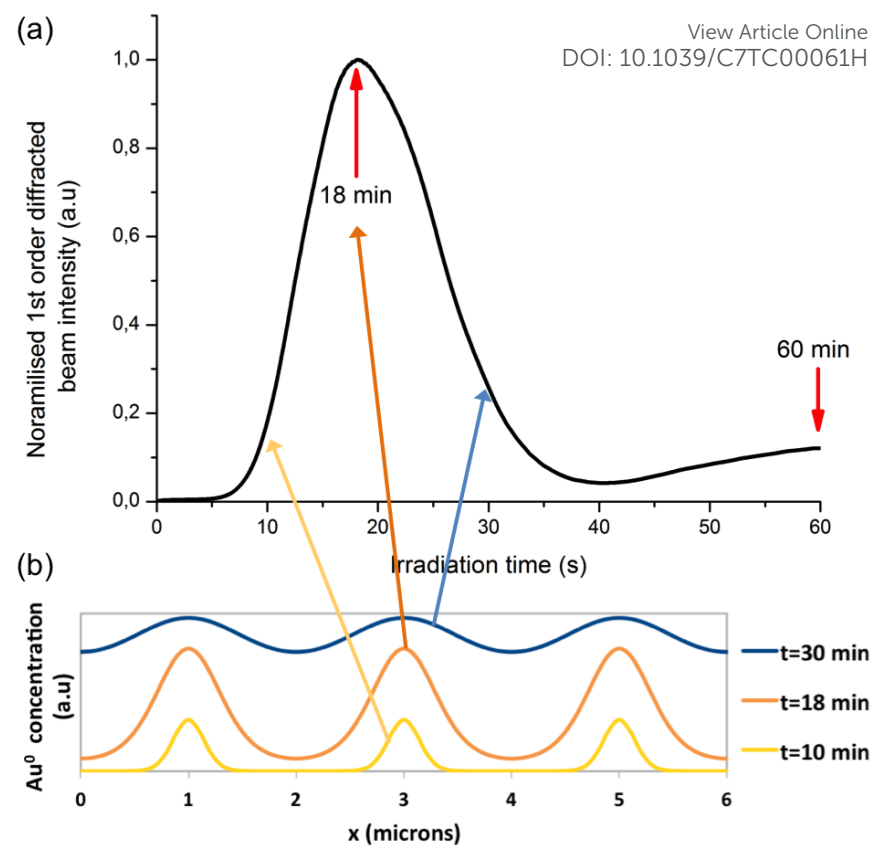

Figure 5: (a) Profile of the first order-diffracted intensity over time during laser irradiation. The maximum of diffraction is reached after $18 \mathrm{~min}$ of irradiation. (b) Schematics of the corresponding gold atoms concentration after 10, 18 and $30 \mathrm{~min}$ of irradiation.

This induces another type of periodic modulation of the refractive index, thus causing diffraction. This hypothesis has been confirmed by other studies on similar samples that have been irradiated for several hours. In this case, the diffraction continues to increase and a peak at around $550 \mathrm{~nm}$ appears in the extinction spectrum of the sample, indicating the presence of gold nanoparticles.

With the aim to investigate the effect of laser intensity, we have varied the flux density in the range $50-150 \mathrm{~mW} / \mathrm{cm}^{2}$. We have observed that photo-reduction kinetics is faster at higher intensity but no significant changes are observed in the structural and optical properties of the nanoparticle gratings obtained after annealing.

To summarize, after $18 \mathrm{~min}$ of irradiation, the sample presents the optimal modulation of the refractive index, with gold atoms in the bright fringes and gold ions in the dark ones. With further irradiation, the variation of the refractive index is reduced as the gold ions are reduced after about $40 \mathrm{~min}$. At this point, the film is almost homogeneous regarding its distribution of the atoms and hence its refractive index. After $60 \mathrm{~min}$ of irradiation, all gold ions are reduced and the refractive index modulation is primarily due to the presence of gold clusters formed preferentially in the bright fringes.

For comparison, we note that the pure PVA films do not show any diffraction or surface structuring under identical irradiations. Finally, it should be emphasized that after $18 \mathrm{~min}$ or $60 \mathrm{~min}$ of irradiation, the diffraction is relatively weak and is only induced by a spatial modulation of the refractive index. Indeed, AFM images of the sample surface at this stage (before annealing) do not show any grating formation. 

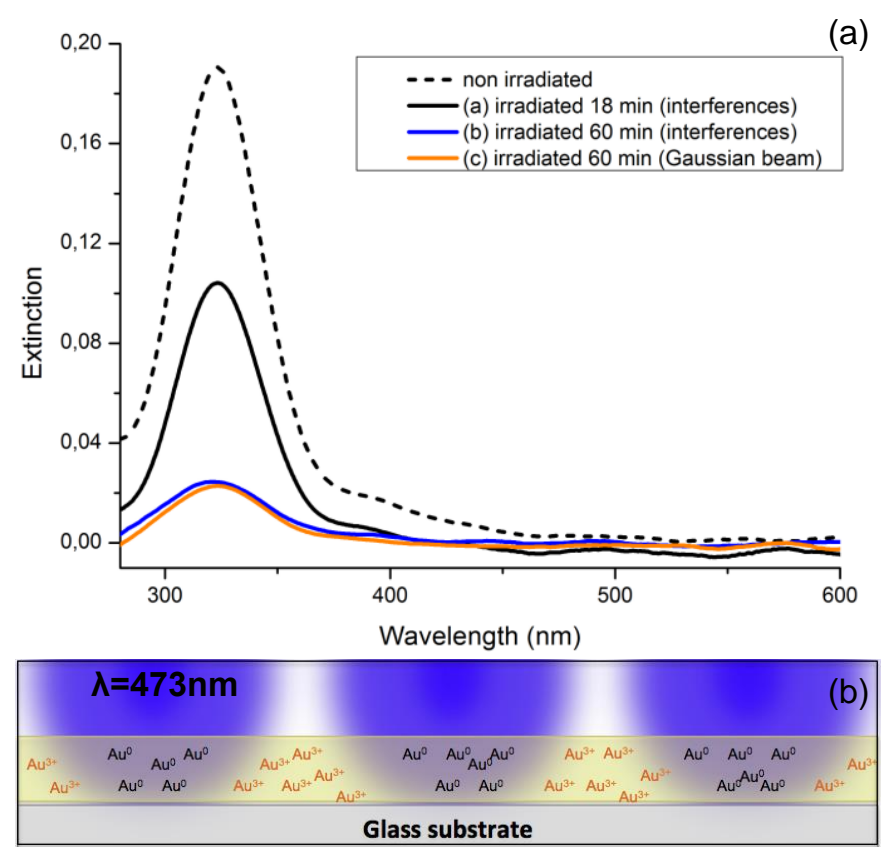

Figure 6: (a) Absorption spectra of a 5\%w sample before irradiation and after $18 \mathrm{~min}$ and $60 \mathrm{~min}$ of irradiation. The spectrum of the same sample irradiated for $60 \mathrm{~min}$ using a gaussian laser beam with the same power is also given for comparison. (b) Schematics of the local photoreduction in the polymer film induced by laser irradiation.

The samples are then annealed as described in Section 2. During annealing, a purple/red color appears in the three irradiated areas (a), (b) and (c), previously defined. The extinction spectra of these areas after annealing show a plasmonic peak thus confirming the presence of nanoparticles. AFM images of the different areas of the sample surface after annealing are given in Fig. 7. In the case of photo-reduction induced by a laser interference pattern (see Fig. 7 (a) and (b)), we observe the formation of surface relief gratings. The amplitude and shape of the gratings is very similar in both cases (a) and (b). The grating height is approximately $100 \mathrm{~nm}$ and the period is $2 \mu \mathrm{m}$, according to the irradiation interference pattern, see Fig. 7.(d). In area (a), where the sample is irradiated for 18 $\mathrm{min}$, the grating profile is very regular, but no nanoparticles are visible on the surface. However, since AFM only probes the surface of the sample, it is difficult to check for the presence of nanoparticles embedded within the polymer. In area (b), which has been exposed for $60 \mathrm{~min}$, we observe the appearance of gold nanoparticles at the surface of the peak regions, where the surface was exposed to the laser irradiation. The nanoparticles size distribution is broad and centered around $50 \mathrm{~nm}$. Finally concerning area (c), which has been irradiated with a homogeneous Gaussian beam, we do not observe any surface relief grating but we do observe the formation of uniformly distributed gold nanoparticles with an average diameter around $30 \mathrm{~nm}$. AFM measurements are not entirely satisfactory in regards to the characterization of the nanoparticle size, shape and spatial distribution.

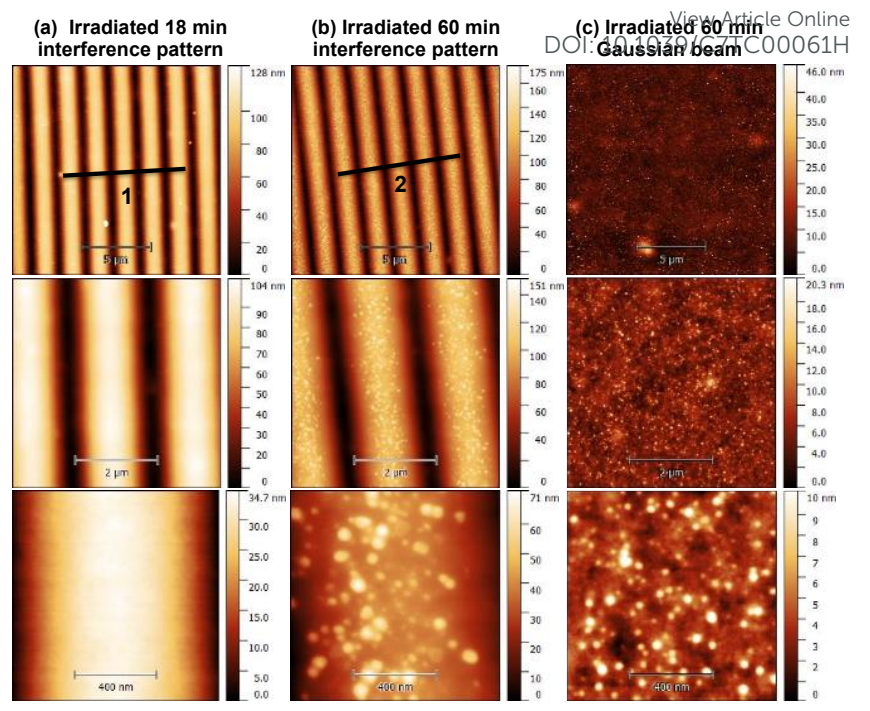

(d) Surface profiles of (a) and (b)

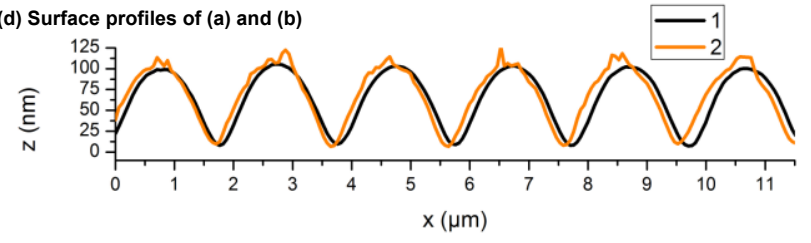

Figure 7: Images (a), (b) and (c) are AFM images of the different areas of the sample surface after annealing, respectively irradiated for 18 and $60 \mathrm{~min}$ with an interference pattern, and $60 \mathrm{~min}$ with a Gaussian beam. From top to bottom, the scale bars are $5 \mu \mathrm{m}$, $2 \mu \mathrm{m}$ and $400 \mathrm{~nm}$. (d) Surface profile corresponding to images (a) and (b).

For this purpose, we have tried to perform SEM measurements of the gratings surface and their cross-sections, however, without much success thus far. The main reason is that SEM imaging is rather complex when dealing with dielectric media deposited on to glass substrates. To address these issues we aim to study the nanoparticle formation using Si substrates.

In the meantime, in order to investigate the presence of embedded nanoparticles under the surface, in each case (a), (b) and (c), we measured bright-field microscope images on localised areas with a diameter of 60 microns. The field depth of the microscope objective is larger than the film thickness, which allows for probing the presence of reflecting objects through the total thickness of the sample. We also measured the extinction spectrum of the corresponding areas. The microscope images and the spectra are measured using the setup described in Section 2. The results are shown in Fig. 8. As it can be seen, the contrast between bright and dark fringes is conserved for areas (a) and (b). The extinction spectra show a plasmonic peak at 500-650 nm, indicating the presence of gold nanoparticles. These results corroborate the presence of nanoparticles embedded in the film, with a higher density in the bright fringes. In case (c), however, no grating is formed and there is no apparent contrast, thus confirming that the nanoparticles are homogeneously distributed in the sample. Regarding the extinction spectra, the sample (c), which presents a uniform distribution of nanoparticles, exhibits the characteristic plasmon resonance peak of gold, though slightly red-shifted, as compared to an individual spherical gold nanoparticle in water. 


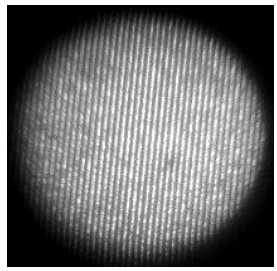

(a)

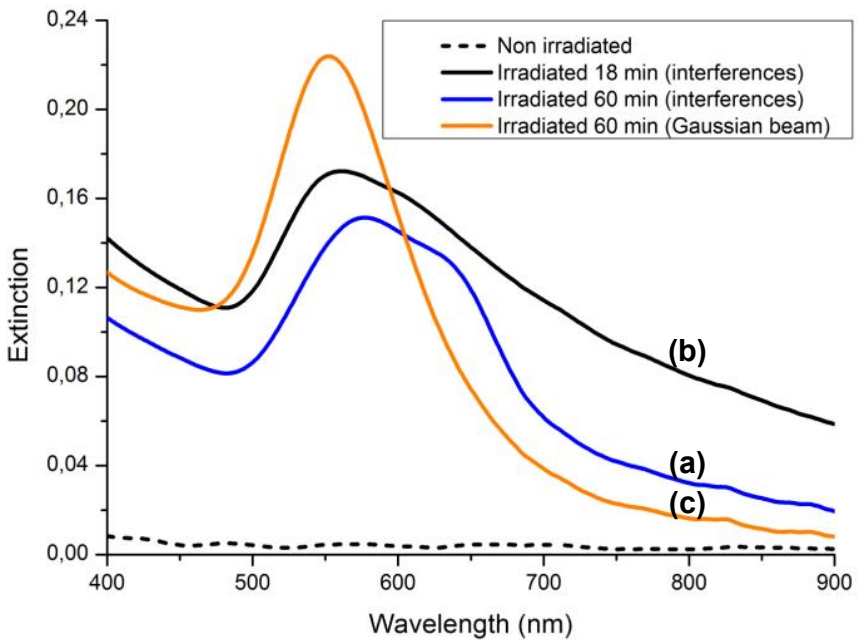

Figure 8: Bright-field images and corresponding extinction spectra of the samples after annealing in the different areas: non irradiated (dashed curve), (a) and (b) irradiated respectively $18 \mathrm{~min}$ and $60 \mathrm{~min}$ with an interference pattern and (c) irradiated $60 \mathrm{~min}$ with a Gaussian beam

This red-shift can be explained by the high refractive index of the surrounding medium ( $\left.\mathrm{n}_{\mathrm{PVA}}=1.5\right)$ and inter-particle interactions ${ }^{29}$. On the other hand, for the samples with gratings, i.e. samples (a) and (b), in addition to being red-shifted, the main resonance peak is damped and broadened. Furthermore, additional peaks appear at $600 \mathrm{~nm}$ and $630 \mathrm{~nm}$, respectively. These peaks may be attributed to new resonance modes induced by the now stronger interactions between nanoparticles, which are organized and more closely packed along the grating crests. Similar effects have been observed on nanoparticles monolayers of different concentrations ${ }^{30}$.

The formation of the (sinusoidal) surface gratings can be attributed to the localized formation of gold nanoparticles. In case (a), these nanoparticles are preferentially formed from the photo-reduced gold atoms located in the bright fringes and therefore follow their spatial distribution. Since the photoreduction is not complete and there are still gold ions in the dark fringes, they are reduced by annealing [15] and are likely to diffuse toward the growing nanoparticles through a ripeninglike process. Therefore, they also participate in the surface structuring. The grating formation mechanism during annealing in this case is summarized in Fig. 9. A similar mechanism takes place in case (b), the difference being that the preferential growth sites here are the gold clusters that were formed after 60 min of irradiation. We may assume that the gold clusters act as seeds and tend to effectively drag the surrounding gold atoms and contribute to the growth of nanoparticles. This may explain the fact that in this situation, annealing $\backslash$ leads to the formation of larger nanoparticles close tothel supface? TC00061H

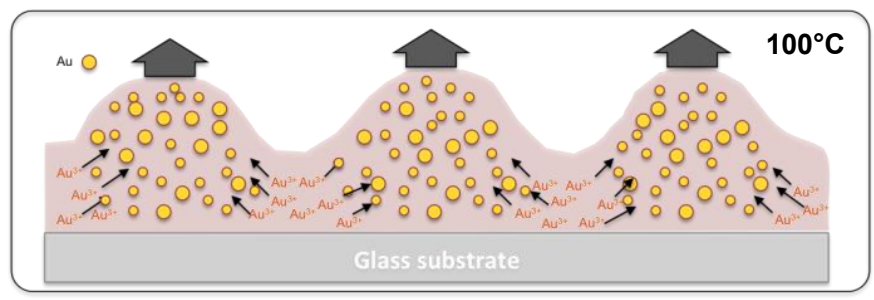

Figure 9: Representation of the effect of annealing in the case (a). The nanoparticles grow from reduced gold atoms as well as from the remaining gold ions that diffuse toward the nanoparticles while simultaneously experiencing thermal reduction. This growth induces surface modulation.

Finally, we have studied the diffractive properties of the gold nanoparticles gratings. We have measured the spectral dependence of the diffraction by taking transmittance spectra at different angles, as explained in Section 2 (see Fig. 4).

The spectra are normalized by the transmitted spectra of the zero order such that the measured diffraction efficiency is defined by

$$
\eta(\alpha, \lambda)=\frac{I_{i}(\alpha, \lambda)}{I_{0}(0, \lambda)}
$$

where $I_{i}(\alpha, \lambda)$ is the transmitted intensity of the ith order, $I_{0}(0, \lambda)$ the transmitted intensity of the zeroth order, $\alpha$ the angle at which the measurement is performed and $\lambda$ is the wavelength of light. Considering that the sample is irradiated at normal incidence, the angular dependence of the diffraction is given by the grating formula

$$
\sin (\alpha)=m \frac{\lambda}{\Lambda}(3)
$$

where $\Lambda$ is the grating period, $m$ an integer that defines the diffracted order and $\alpha$ the angle at which the wavelength will be diffracted in this order. The diffraction efficiency maps are given in Fig. 10. The measurement is focused on the $1^{\text {st }}$ and $2^{\text {nd }}$ diffracted orders and the slope we measure for the $1^{\text {st }}$ order is in good agreement with Eq. (3). We can clearly see a resonant profile in the diffracted orders between $550 \mathrm{~nm}$ and $700 \mathrm{~nm}$ for both samples. If we now look closer at the diffracted efficiency magnitude of the $1^{\text {st }}$ order, we can plot the maximum value of diffraction efficiency as a function of wavelength. We obtain the diffraction efficiency spectra (see graphs below diffraction efficiency maps in Fig. 10, black curve). These spectra clearly exhibit plasmon resonance profiles that are very similar to the extinction spectra we measured for this sample (see Fig. 8). This effect, called plasmon-enhanced diffraction efficiency, is attributed to the plasmonic nanoparticles present in the film. A similar behaviour has only been reported recently in the literature ${ }^{23}$, but never with such a spectral matching between plasmonic properties and diffraction efficiency that is observed here. We note that regardless of the spectral profile matching, it seems that the diffraction efficiency is higher in the case where nanoparticles are present at the film surface. 

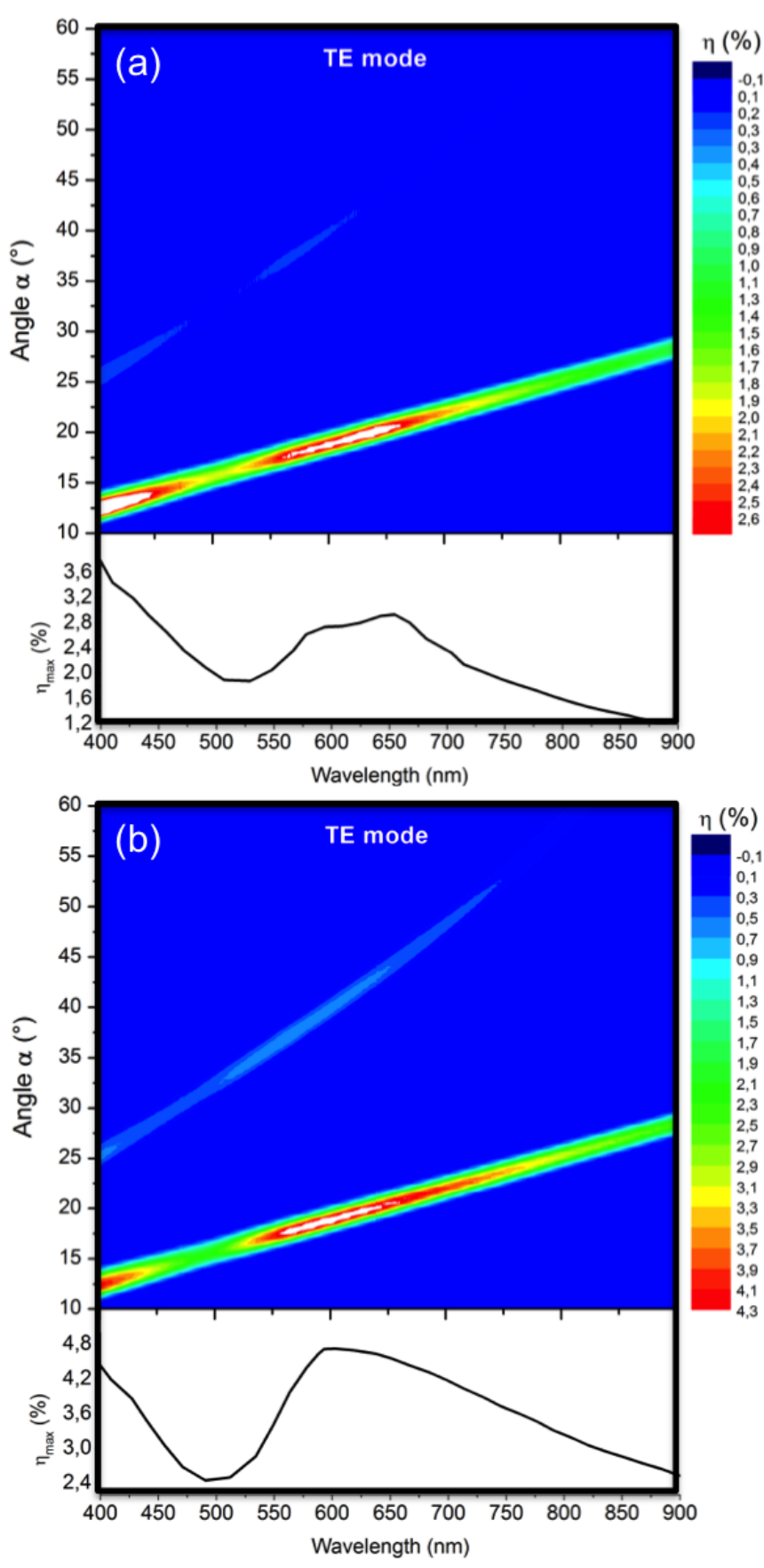

Figure 10: Diffraction efficiency maps showing the spectral dependence of the gratings (a) and (b) irradiated respectively for $18 \mathrm{~min}$ and $60 \mathrm{~min}$ with an interference pattern. The measurement has been performed for the $1^{\text {st }}$ and $2^{\mathrm{d}}$ order-diffracted beams. The graphs below the diffraction efficiency maps show the corresponding maximum of diffraction efficiency as a function of wavelength.

The amplitude of the diffraction efficiency measured here, which reaches $4.8 \%$ at the plasmon resonance, is relatively high. Indeed, the maximum theoretical diffraction efficiency of the $1^{\text {st }}$ order for a thin sinusoidal transmission grating is 6.25 $\%^{31}$. Considering that the amplitude of our grating is only 100 $\mathrm{nm}$, an efficiency of $4.8 \%$ is unexpected and can only be explained by the plasmonic enhancement due to the presence of gold nanoparticles. A possible interpretation of this plasmon- induced enhancement is that the energy absorbed $d_{\text {tic }}$ by the

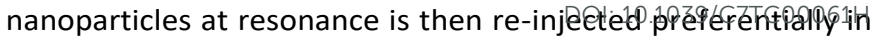
the diffracted orders, which then enhances the diffraction efficiency. We would like to emphasize that all measurements presented here have been repeated 3 months after fabrication and no change was observed. This attests to the good stability of our PNG over time.

\section{Conclusions}

We have shown that it is possible to synthesize structured plasmonic nanoparticle composite films by using in situ interferometric photo-reduction of gold precursors in a polymer matrix with visible light followed by an annealing procedure. Indeed, the spatially controlled photo-reduction leads to the formation of surface relief gratings induced during nanoparticle growth. Our measurments have shown that the concommitant effects of i) periodic surface and refractive index modulation and ii) plasmonic properties of the nanoparticles, lead to the phenomenon of plasmon-enhanced diffraction efficiency. More precisely, the plasmonic nanoparticle gratings we have synthesized present a significant enhancement of the diffraction efficiency in the region of the plasmon resonance of gold nanoparticles.

The fabrication approach we propose here is based on low cost and environment-friendly materials and involves only simple experimental techniques. In addition, the laser patterning technique used here is quite suitable for large-scale synthesis of organized nanostructures. In particular, it is possible to design diffractive optical materials with tailored dispersion and plasmon-enhanced diffraction efficiencies.

However, a few issues still require further investigation. For instance, the photo-reduction mechanism that occurs in the visible range has to be described in more detail. In particular, gold(I) intermediates might be involved. In addition, a systematic study of experimental parameters such as gold concentration, film thickness and irradiation power, is needed to better understand the principles underlying the gratings formation. Finally, theoretical developments are in progress with the aim to model such systems and compute their optical properties. More precisely, we use the Rigorous Coupled-Wave Analysis method, together with the effective-medium approach, to study the diffraction efficiency of plasmonic nanoparticle gratings.

\section{Acknowledgements}

We would like to acknowledge financial support from the University of Perpignan and the laboratory PROMES. We would also like to thank the PPCM team for giving us access to their experimental facilities.

\section{References}

1 A. Kaushik, R. Kumar, S. K. Arya, M. Nair, B. D. Malhotra and S. Bhansali, Chem. Rev., 2015, 115, 4571-4606. 
Sudheer, P. Tiwari, S. Bhartiya, C. Mukherjee, M. N. Singh, A. K. Sinha, V. N. Rai and A. K. Srivastava, J. Appl. Phys., 2015, 118, 64303. M. K. Hedayati, F. Faupel and M. Elbahri, Materials (Basel). 2014, 7, 1221-1248. X. Chen, L. Zuo, W. Fu, Q. Yan, C. Fan and H. Chen, Sol. Energy Mater. Sol. Cells, 2013, 111, 1-8. E. Stratakis and E. Kymakis, Mater. Today, 2013, 16, 133146.

R. C. Bailey, J. Nam, C. A. Mirkin and J. T. Hupp, Journals Am. Soc., 2003, 125, 13541-13547.

A. W. Wark, H. J. Lee, A. J. Qavi and R. M. Corn, 2007, 79, 6697-6701.

W. H. Yeh, J. W. Petefish and A. C. Hillier, Anal. Chem., 2011, 83, 6047-6053.

K. G. Yager and C. J. Barrett, Macromolecules, 2006, 39 9320-9326.

Y. Tomita, in Photorefractive Effects, Materials, and Devices, ed. G. . K. D. . N. D. . and X. J. Zhang, Optical Society of America, Sanya, Hainan, China, 2005, vol. 99, p. 274.

A. T. Juhl, J. D. Busbee, J. J. Koval, L. V Natarajan, V. P. Tondiglia, R. A. Vaia, T. J. Bunning and P. V Braun, ACS Nano, 2010, 4, 5953-61.

D. Nazarova, L. Nedelchev, P. Sharlandjiev and V. Dragostinova, Appl. Opt., 2013, 52, E28-33. S. K. Na; J. S. Kim; C. H. Oh;, J. Korean Phys.Soc., 2008, 53, 2316-2319.

S. Porel, S. Singh and T. P. Radhakrishnan, Chem. Commun (Camb)., 2005, 2387-2389.

A. Pucci, M. Bernabò, P. Elvati, L. I. Meza, F. Galembeck, C. A. de Paula Leite, N. Tirelli and G. Ruggeri, J. Mater. Chem., 2006, 16, 1058.

B. Kang and J. W. Wu, J. Korean Phys. Soc., 2006, 49, 955958.

S. Porel, N. Venkatram, D. N. Rao and T. P. Radhakrishnan, J. Nanosci. Nanotechnol., 2007, 7, 1887-92.

T. Kunze, T. Roch, G. Anne, A. Rank, G. Denise and M. Bieda, SPIE Newsroom, 2016, 2-4.

K. Kaneko, H.-B. Sun, X.-M. Duan and S. Kawata, Appl. Phys. Lett., 2003, 83, 1426.

T. Ritacco, L. Ricciardi, M. La Deda and M. Giocondo, J. Eur. Opt. Soc.-Rapid, 2016, 11. M. Rumi and J. W. Perry, Adv. Opt. Photonics, 2010, 2, 451.

Y. Lin, X. Zhang, S. Bai and A. Hu, J. Mater. Chem. C, 2015, 3, 6046-6052.

Srivastava, T. K. Sharma, V. N. Rai, A. K. Srivastava and P. A. Naik, J. Appl. Phys., 2016, 120.

K. Yager and C. Barrett, in Polymeric Nanostructures and Their Applications, 2006, pp. 1-38. DOI: 10.1039/C7TC00061H

A. K. Gangopadhayay and A. Chakravorty, J. Chem. Phys., 1961, 35, 2206-2209.

M. K. Abyaneh, D. Paramanik, S. Varma, S. W. Gosavi and S. K. Kulkarni, J. Phys. D. Appl. Phys., 2007, 40, 3771-3779.

G. L. Nealon, B. Donnio, R. Greget, J.-P. Kappler, E. Terazzi and J.-L. Gallani, Nanoscale, 2012, 4, 5244-58.

E. Yilmaz, G. Ertas, E. Bengu and S. Suzer, J. Phys. Chem. C, 2010, 114, 18401-18406.

C. Noguez, J. Phys. Chem. C, 2007, 111, 3806-3819.

C. Jiang, S. Markutsya and V. V. Tsukruk, Langmuir, 2004, 20, 882-890.

R. Magnusson and T. K. Gaylord, Opt. Commun., 1979, 28, 1-3. 
This is a novel method for fabricating plasmonic nanoparticle gratings with original optical properties induced by laser photo-reduction of gold precursors in PVA thin films.

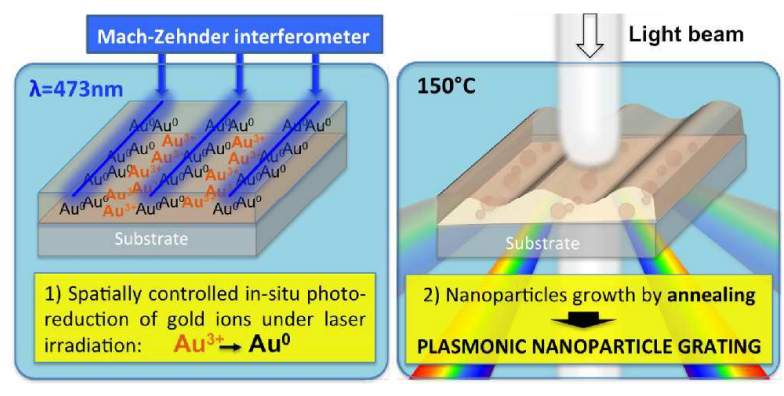

